\title{
Results from single shot grazing incidence hard $x$-ray damage measurements conducted at the SACLA FEL
}

\author{
Andrew Aquila ${ }^{\mathrm{a}}$, Cigdem Ozkan ${ }^{\mathrm{a}}$, Ryszard Sobierajski ${ }^{\mathrm{b}}$, Vera Hájkovác ${ }^{\mathrm{c}}$, Tomás Burian ${ }^{\mathrm{c}}$, Jaromír \\ Chalupsky ${ }^{\mathrm{c}}$, Libor Juha ${ }^{\mathrm{c}}$, Michael Störmer ${ }^{\mathrm{d}}$, Haruhiko Ohashi ${ }^{\mathrm{e}, \mathrm{f}}$, Takahisa Koyama ${ }^{\mathrm{e}}$, Kensuke Tono ${ }^{\mathrm{e}}$, \\ Yuichi Inubushi ${ }^{\mathrm{f}}$, Makina Yabashif ${ }^{\mathrm{f}}$, Harald Sinn ${ }^{\mathrm{a}}$, Thomas Tschentscher ${ }^{\mathrm{a}}$, Adrian P . Mancuso ${ }^{\mathrm{a}}$, \\ Jérôme Gaudin ${ }^{\mathrm{g}}$ \\ ${ }^{a}$ European XFEL (Germany); ${ }^{b}$ Institute of Physics (Poland); ${ }^{\circ}$ Institute of Physics of the ASCR, v.v.i. \\ (Czech Republic); ${ }^{\mathrm{d} H e l m h o l t z-Z e n t r u m ~ G e e s t h a c h t ~(G e r m a n y) ; ~}{ }^{\mathrm{e} J a p a n ~ S y n c h r o t r o n ~ R a d i a t i o n ~}$ \\ Research Institute (Japan); ${ }^{\mathrm{f}}$ RIKEN (Japan) ${ }^{\mathrm{g}}$ University of Bordeaux (France)
}

\begin{abstract}
With the development of hard X-ray free electron lasers, there is a pressing need to experimentally determine the single shot damage limits of presently used and potential future optical coating materials. To this end we present damage results, and analysis of fluence threshold limits, from grazing incidence geometry experiments conducted at the Spring-8 Angstrom Compact free electron LAser (SACLA) on Carbon coatings at 7 and $12 \mathrm{keV}$ photon energies.
\end{abstract}

Keywords: X-ray mirrors, damage results, carbon coatings

\section{INTRODUCTION}

Hard X-ray Free Electron Lasers (XFELs) open the possibilities for experiments that would be impractical or impossible at other X-ray facilities such as synchrotrons. These include, but are not limited to, experiments on 3D single particle diffraction imaging ${ }^{1}$ the dynamics of biologic crystals ${ }^{2}$, and non-linear experiments ${ }^{3}$ with X-rays. With more XFEL facilities being built and planned, it is foreseen that they will aim to deliver larger pulse energies and higher peak brilliances. With the desire to increase in pulse power the natural question arises: what is the maximum pulse fluence that a grazing incidence FEL mirror can tolerate? To facilitate the answer to this question, we have conducted grazing incidence damage studies at the Spring-8 Angstrom Compact free electron LAser (SACLA) facility on Carbon coated mirrors at 7 and $12 \mathrm{keV}$ photon energies.

We choose Carbon as a mirror material due to its current use in Soft X-ray FELs, the extensive work on optical damage of amorphous carbon at lower energies ${ }^{4-10}$, and as its low $\mathrm{Z}$ nature, small complex part of its index of refraction, dissipates the absorbed energy over a larger volume.

\section{EXPERIMENTAL SETUP}

The experiment was conducted at the SACLA X-ray FEL user facility ${ }^{11}$. The facility provides X-ray pulses with an average pulse energy of order $200 \mu \mathrm{J}$ at $7 \mathrm{keV}$ photon energies and slightly less at $12 \mathrm{keV}$. A pair of Kirkpatrick-Baez (KB) optics was used to focus the beam to a spot size of $1 \mu \mathrm{m}$ by $1 \mu \mathrm{m}$ full width at half maximum (FWHM) measured with a knife-edge wire scan ${ }^{12}$. The system efficiency, including Beryllium isolation valves, was of the order of $50 \%$, meaning at both 7 and $12 \mathrm{keV}$ a maximum deliverable pulse energy, to the test mirror sample, was $100 \mu \mathrm{J}$.

The test mirror sample is a super polished single crystal silicon optic $100 \mathrm{~mm}$ long, $10 \mathrm{~mm}$ wide and $8 \mathrm{~mm}$ thick, coated with an amorphous carbon layer $50.1 \mathrm{~nm}$ thick using DC magnetron sputtering. The density of the coating was slightly less than bulk density of $2.2 \mathrm{~g} / \mathrm{cm}^{3}$. Furthermore root mean squared roughness of the mirror substrate was estimated to be $0.7 \mathrm{~nm}$ using Copper K-alpha reflectometry.

During the experiment we used procedures and techniques used in recent experiments ${ }^{13}$ at the Free-Electron LASer in Hamburg (FLASH) facility. The sample was measured at grazing angles typically used for single coated grazing X-ray optics. The mirror sample was placed in the focal position of the beamline KB optics for alignment and measurement. An alignment camera was located $2 \mathrm{~m}$ downstream of the sample and registered the position of either direct beam or the

Damage to VUV, EUV, and X-ray Optics IV; and EUV and X-ray Optics: Synergy between Laboratory and Space III, edited by Libor Juha, Saša Bajt, Richard London, René Hudec, Ladislav Pina, Proc. of SPIE Vol. 8777, 87770H

(C) 2013 SPIE · CCC code: $0277-786 X / 13 / \$ 18 \cdot$ doi: $10.1117 / 12.2017725$ 
beam reflected from the sample. Using an attenuated XFEL beam we aligned the optical sample to an angular tolerance of better than $0.05 \mathrm{mrad}$.

For the $7 \mathrm{keV}$ measurements a sample angle of $4 \mathrm{mrad}$ was chosen. This angle is below the critical angle at $7 \mathrm{keV}$. We collected 60 single shot damage measurements under various fluence conditions. We used the full XFEL beam, unattenuated, along with 3 different levels of attenuation using 25, 50 and $75 \mu \mathrm{m}$ thick Aluminum foils. The incident pulse energy for each damage measurement was recorded using a gas intensity monitor. The shot-to-shot pulse energy jitter was measured to be $9 \%$ at $7 \mathrm{keV}$ and $16 \%$ at $12 \mathrm{keV}$. This jitter allowed for a near continuous change in power settings between the discrete attenuation settings.

For the $12 \mathrm{keV}$ measurements the sample angle was first chosen to be $2 \mathrm{mrad}$, which is below the critical angle at 12 $\mathrm{keV}$. However, the full XFEL beam left no observable damage to the sample. One hundred full fluence, $100 \mu \mathrm{J}$ pulses were collected in the same location on the sample. This was collected with the notion that any damage, even if it was too small to be observed, would accumulate and show an effect. However, this proved not to be the case. We concluded that there was not enough pulse energy required to damage the sample below the critical angle using the $1 \mu \mathrm{m}$ by $1 \mu \mathrm{m}$ FWHM XFEL beam.

Measurements were also conducted at $3.5 \mathrm{mrad}$ angle, which is above the critical angle. The information gathered at angles above the critical angle, where the sample goes from reflecting $>99 \%$ of the incident pulse energy to $<1 \%$, is important for alignment consideration. If a beamline mirror for an XFEL becomes misaligned, it is critical to know the damage threshold under the worst-case scenario. Twenty-five damage measurements were taken above the critical angle under 3 different attenuation settings using a combination of Silicon and Aluminum filters. A representative set of damage craters is shown in Figure 1.
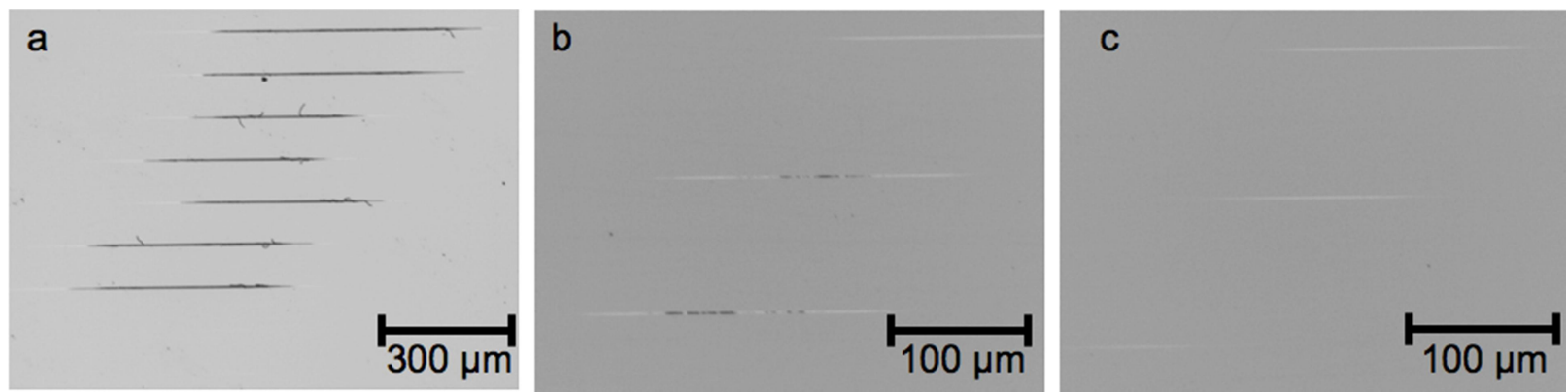

Figure 1: Representative confocal microscopy of the single shot damage imprint craters. (a) $12 \mathrm{keV}$ photons energy with an average pulse energy of $35 \mu \mathrm{J}$ at $3.5 \mathrm{mrad}$ incidence angle. (b) $7 \mathrm{keV}$ photon energy with an average pulse energy of 75 $\mu \mathrm{J}$ at $4.0 \mathrm{mrad}$ incidence angle. (c) $7 \mathrm{keV}$ photon energy with an average pulse energy of $48 \mu \mathbf{J}$ at $4.0 \mathrm{mrad}$ incidence angle.

\section{ANALYSIS AND RESULTS}

To determine the threshold damage fluence, we used the method of fitting the area of the damage spot size to the logarithm of the pulse energy ${ }^{14}$. The intercept of the linear fit determines the threshold pulse energy below which no single shot damage should be observable. This method relies on the assumption of an elliptically Gaussian incident beam. The lengths of the damage craters were determined using confocal microscopy. Scanning electron microscopy and atomic force microscopy were both tried, however due to their aspect ratio varying from $50 \mu \mathrm{m}$ to $1 \mathrm{~mm}$ in length and $<1 \mu \mathrm{m}$ in width, these methods prove impractical to determine the area of the damage craters. Confocal microscopy also proved challenging, as the resolution of the images could not provide accurate determination of the widths of each of the damage craters. To approximate the widths, and hence the areas, it is noted that the knife-edge beam profile scan of the cross-section are nearly equal in size horizontally and vertically. Therefore a reasonable estimate of the width is the projection of the length. In other words, the width is set equal to the sine of the grazing angle multiplied by the length.

A plot of the damage area against the logarithm of the pulse energy is shown in Figure 2. Fitting the pulse energy to damage area yields a threshold damage pulse energy of $38.4 \mu \mathrm{J}$, for the $7 \mathrm{keV}$ photon energy at 4 mrad. For the $12 \mathrm{keV}$ data a pulse energy of $5.2 \mu \mathrm{J}$ was determined to damage the optic above the critical angle at $3.5 \mathrm{mrad}$, and a pulse energy 
above $100 \mu \mathrm{J}$ is required to damage the optic below the critical angle at $2 \mathrm{mrad}$. If we use the $1 \mu \mathrm{m} \times 1 \mu \mathrm{m}$ FWHM focal spot size then the single pulse damage fluence limit at $7 \mathrm{keV}$ becomes $3.84 \mathrm{~kJ} / \mathrm{cm}^{2}$ and at $12 \mathrm{keV}$ become $10 \mathrm{~kJ} / \mathrm{cm}^{2}$ below the critical angle and $0.5 \mathrm{~kJ} / \mathrm{cm}^{2}$ above the critical angle. The values below the critical angle may appear to be high, but recall that nearly all the pulse energy is reflected off the mirror.

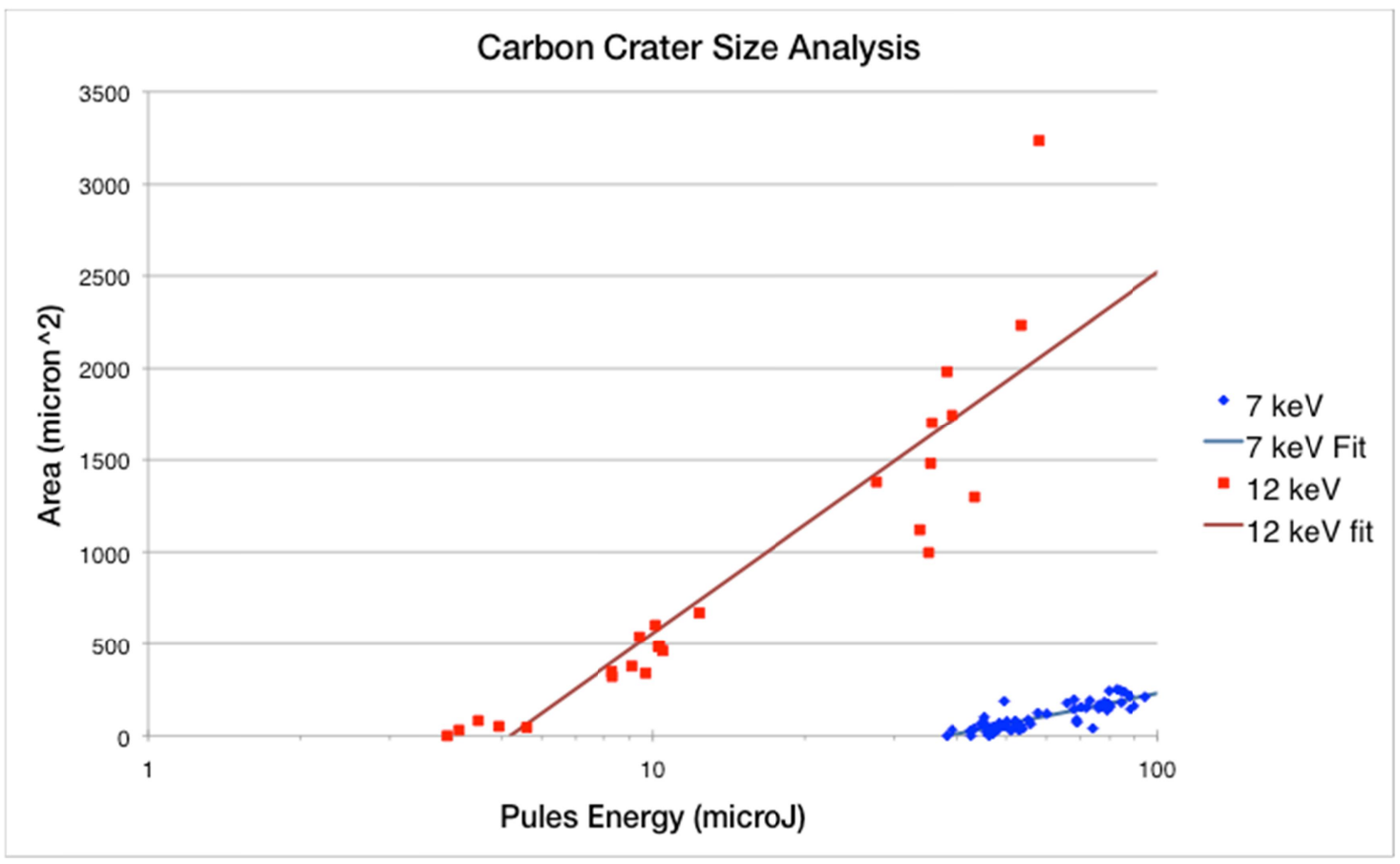

Figure 2: Damaged area verses logarithm of pulse energy with fits for $7 \mathrm{keV}$ and $12 \mathrm{keV}$.

\section{CONCLUSION}

We have conducted single shot damage measurements on amorphous Carbon to determine damage fluence thresholds for grazing incidence XFEL optics. At $7 \mathrm{keV}$ with a $4 \mathrm{mrad}$ grazing angle, just below the critical angle, the damage fluence was determined to be $38.4 \mathrm{~J} / \mathrm{cm}^{2}$. For $12 \mathrm{keV}$ the threshold damage fluence was determined to be grater then $10 \mathrm{~kJ} / \mathrm{cm}^{2}$ below the critical angle and $5 \mathrm{~J} / \mathrm{cm}^{2}$ just above the critical angle.

\section{REFERENCES}

[1] Neutze, R. et al, "Potential for biomolecular imaging with femtosecond X-ray pulses," Nature 406, 752-757 (2000)

[2] Aquila, A. et al, "Time-resolved protein nanocrystallography using an X-ray free-electron laser," Optics Express 20, 2706-2716 (2012)

[3] Nagler, B. et al, "Turning solid aluminium transparent by intense soft X-ray photoionization," Nature Physics 5, 693 - 696 (2009)

[4] Gaudin, J. et al, "Investigating the interaction of x-ray free electron laser radiation with grating structure," Optics Letters 37, 3033-3035 (2012)

[5] Gaudin, J. et al, "Amorphous to crystalline phase transition in carbon induced by intense femtosecond x-ray free-electron laser pulses," Phys. Rev. B 86, 024103 (2012)

[6] Hau-Riege, S. P. et al, "Ultrafast Transitions from Solid to Liquid and Plasma States of Graphite Induced by XRay Free-Electron Laser Pulses,” Phys. Rev. Lett. 108, 217402 (2012)

[7] Hau-Riege, S. P. et al, "Wavelength dependence of the damage threshold of inorganic materials under extremeultraviolet free-electron-laser irradiation," Appl. Phys. Lett. 95, 111104 (2009) 
[8] Chalupský, J. et al, "Damage of amorphous carbon induced by soft x-ray femtosecond pulses above and below the critical angle," Appl. Phys. Lett. 95, 031111 (2009)

[9] Hau-Riege, S. P. et al, "Damage threshold of inorganic solids under free-electron-laser irradiation at $32.5 \mathrm{~nm}$ wavelength," Appl. Phys. Lett. 90, 173128 (2007)

[10] Hau-Riege, S. P. et al, "Subnanometer-Scale Measurements of the Interaction of Ultrafast Soft X-Ray FreeElectron-Laser Pulses with Matter," Phys. Rev. Lett. 98, 145502 (2007)

[11] Ishikawa, T. et al, "A compact X-ray free-electron laser emitting in the sub-ångström region,” Nature Photonics 6, 540-544 (2012)

[12] Yumoto, H. et al, "Focusing of X-ray free-electron laser pulses with reflective optics," Nature Photonics 7, 4347 (2013)

[13] Sobierajski, R. et al., "Experimental set-up and procedures for the investigation of XUV free electron laser interactions with solids," Journal of Instrumentation 8, P02010 (2013)

[14] Liu, J. M, "Simple technique for measurements of pulsed Gaussian-beam spot sizes," Optics Letters, 7, 196-198 (1982) 